\title{
Compaixão e medicina centrada na pessoa: convergências entre o Dalai Lama Tenzin Gyatso e lan McWhinney
}

\author{
Compassion and patient-centered medicine: convergences between the Dalai Lama Tenzin \\ Gyatso and lan McWhinney
}

\section{La compasión y la medicina centrada en la persona: convergencias entre el Dalai Lama Tenzin Gyatso y lan McWhinney}

Leandro David Wenceslau. Departamento de Medicina e Enfermagem, Universidade Federal de Viçosa (UFV). Viçosa, MG, Brasil. leandro.david@ufv.br (Autor correspondente)

Eberhart Portocarrero-Gross. Clínica da Família Maria do Socorro Silva e Souza, Secretaria Municipal de Saúde do

Rio de Janeiro. Rio de Janeiro, RJ, Brasil. eberhartpg@ gmail.com

Marcelo Marcos Piva Demarzo. Centro Brasileiro de Mindfulness e Promoção da Saúde, Escola Paulista de Medicina, Universidade Federal do Estado de São Paulo (UNIFESP). São Paulo, SP, Brasil. demarzo@ unifesp.br

\section{Resumo}

O método clínico centrado na pessoa (MCCP) é uma proposta de reorientação da prática clínica que identifica um distanciamento, na medicina contemporânea, da experiência subjetiva e qualitativa dos pacientes. Para superar este distanciamento, o MCCP utiliza teorias e técnicas de diversas escolas da psicologia moderna ocidental. Neste trabalho, analisamos possíveis contribuições para o MCCP de uma escola de pensamento oriental, o budismo tibetano, na perspectiva delineada por seu atual líder espiritual, Tenzin Gyatso, o XIV Dalai Lama. Também são utilizados elementos de trabalhos de lan McWhinney para intermediar e enriquecer esta reflexão, que apresenta como proposição final o desenvolvimento da compaixão como qualidade fundamental para lidar com a dimensão psicossocial do sofrimento humano. O MCCP e a abordagem ética budista plasmada no pensamento do Dalai Lama compartilham um objetivo comum: uma compreensão e um manejo não reducionistas do sofrimento humano. Em ambos os casos, identifica-se a necessidade de reconhecer e valorizar aspectos mentais e emocionais das relações humanas como estratégia para lograr esta abordagem ampliada.

\section{Abstract}

The patient-centered clinical method (PCCM) is a proposal of reorienting contemporary medical practice that identifies a distance from the patients' subjective and qualitative experiences. To overcome this gap, the PCCM uses theories and techniques from several schools of modern Western psychology. In this paper, we analyze possible contributions to the PCCM from an Eastern school of thought - Tibetan Buddhism - using the perspective of its current spiritual leader, Tenzin Gyatso, the fourteenth Dalai Lama. Additionally, elements of lan McWhinney's work mediate and enrich this reflection. The development of compassion is the fundamental quality to manage the psychosocial dimension of human suffering. The PCCM and the Buddhist ethical approach, delineated by the Dalai Lama, share a common goal: understanding and non-reductionist management of human suffering. In both cases, we identify recognition and valuing the mental and emotional aspects of human relations as a strategy to achieve this comprehensive approach.

Como citar: Wenceslau LD, Portocarrero-Gross E, Demarzo MMP. Compaixão e medicina centrada na pessoa: convergências entre o Dalai Lama Tenzin Gyatso e lan McWhinney. Rev Bras Med Fam Comunidade. 2016;11(38):1-10. http://dx.doi.org/10.5712/rbmfc11(38)1138
Palavras-chave: Filosofia Médica Ética Budismo Keywords: Philosophy, Medical Ethics Buddhism

Fonte de financiamento: declaram não haver. Parecer CEP: não se aplica. Conflito de interesses: declaram não haver. Procedência e revisão por pares: revisado por pares. Recebido em: 19/05/2015. Aprovado em: 03/02/2016. 


\section{Resumen}

El método clínico centrado en la persona (MCCP) es una propuesta de reorientación de la práctica médica que identifica, en la medicina contemporánea, un distanciamiento de la experiencia subjetiva y cualitativa de los pacientes. Para superar esta limitación, el MCCP utiliza teorías y técnicas de diversas escuelas de psicología occidental moderna. En este trabajo, se analizan posibles contribuciones al MCCP de una escuela de pensamiento oriental, el budismo tibetano, en la perspectiva esbozada por su actual líder espiritual, Tenzin Gyatso, el decimocuarto Dalai Lama. También se utilizan elementos de trabajos de lan McWhinney para mediar y enriquecer esta reflexión que presenta como propuesta final el desarrollo de la compasión como cualidad fundamental para hacer frente a la dimensión psicosocial del sufrimiento humano. EI MCCP y el enfoque ético budista delineado por el Dalai Lama comparten un objetivo común: una comprensión y un tratamiento no reduccionista del sufrimiento humano. En ambos casos, se identifica la necesidad de reconocer y valorar los aspectos mentales y emocionales de las relaciones humanas como una estrategia para lograr este enfoque ampliado.
Palabras clave:

Filosofía Médica

Ética

Budismo

\section{Introdução}

O método clínico centrado na pessoa (MCCP) é um modelo de orientação da prática clínica desenvolvido e validado por pesquisadores do Departamento de Medicina de Família da Universidade de Western Ontario, no Canadá, entre as décadas de 70 e $80 .^{1,2}$ Foi elaborado com o objetivo de traduzir, em orientações práticas, uma teoria médica que reconhece os avanços obtidos pela incorporação do método científico moderno à Medicina, mas que, ao mesmo tempo, identifica um distanciamento da prática médica contemporânea da experiência subjetiva de "sofrimento" que emerge das pessoas que classificamos como "doentes". ${ }^{3}$ Ainda que sua aplicação não esteja restrita à Medicina de Família e Comunidade (MFC), pesquisadores e práticos desta disciplina reconhecem o MCCP como ferramenta fundamental para uma prática clínica em coerência com os princípios que norteiam a especialidade. ${ }^{4,5}$

O método clínico baseado no modelo biomédico, hegemonicamente praticado nos tempos contemporâneos, assume como objeto central da medicina a identificação e a intervenção sobre a doença. ${ }^{6}$ Doenças são entendidas como variações biológicas anormais que podem ser medidas e classificadas em grupos cujas características gerais são estipuladas de forma abstrata. ${ }^{7}$ Assim, aspectos psicossociais do processo de adoecimento são considerados periféricos e de pouca relevância clínica. ${ }^{8}$

O MCCP tem como objetivo corrigir esse desequilíbrio, introduzindo na prática clínica ações e ferramentas para lidar com a experiência da pessoa enferma e para convidá-la a atuar como protagonista da construção e da concretização de um plano de manejo de seus problemas. ${ }^{9}$ Para lograr este objetivo, 0 MCCP, em sua versão mais recente, reúne quatro componentes: explorar a saúde, a doença e a experiência da doença; entender a pessoa como um todo; elaborar um um plano conjunto de manejo de problemas; e intensificar o relacionamento entre pessoa e médico. ${ }^{1,2,10}$

Para operar estes componentes, são sugeridas diversas ferramentas desenvolvidas a partir de referenciais da psicologia moderna, principalmente de escolas da Europa e América do Norte, como a entrevista motivacional, a tomada de decisão compartilhada e informada, a compreensão e uso dos conceitos de transferência e contratransferência advindos da psicanálise, entre outras ferramentas. ${ }^{11-13}$

\section{Diálogos entre budismo e medicina de família e comunidade}

O diálogo entre elementos teóricos e práticos do budismo e da medicina de família, ainda que não seja um tema frequente, já foi abordado em estudos anteriores. Por exemplo, o conceito de metta ou gentileza amorosa é apresentado por Aung ${ }^{14}$ como a contribuição essencial do budismo para a atenção primária e 
a abordagem centrada na pessoa. Neher e Brown ${ }^{15}$ descreveram como alguns princípios pedagógicos do zen budismo podem contribuir com o ensino da medicina de família. Na mesma direção, Garrett et al. ${ }^{16}$ propõem um modelo inspirado nos koans zens para promover o aprendizado de habilidades psicossociais da medicina de família e comunidade.

Neighbour, no capítulo Zen and the art of the consultation de sua obra The inner consultation ${ }^{17}$, descreve como sentimentos e pensamentos, experimentados pelos profissionais nas consultas médicas, podem ser abordados, em sua complexidade e paradoxalidade, a partir da perspectiva zen. Esta perspectiva, segundo Neighbour, inclui três elementos: a meditação, a prática na vida cotidiana e o manejo intuitivo do pensamento "além dos limites do intelecto" (tradução nossa, p. 256).

Por último, destacamos o uso crescente, na atenção primária, de intervenções baseadas em mindfulness (IBM) ${ }^{18} \mathrm{e}$ da terapia de aceitação e compromisso (TAC). ${ }^{19} \mathrm{Em}$ parte, os fundamentos teóricos e práticos destas intervenções são atribuídos ao budismo. Hayes ${ }^{20}$ e Fung ${ }^{21}$ argumentam que temas centrais do budismo como a ubiquidade do sofrimento humano, a importância da atenção plena ou mindfulness e a promoção de ações emocionalmente positivas possuem paralelos claros com a filosofia e tecnologias da TAC. De forma semelhante, as IBM também são, em parte, derivadas de práticas meditativas budistas, embora, no seu processo de ocidentalização, alguns princípios filosóficos budistas não estejam mais presentes nestas abordagens. ${ }^{22}$

Tenzin Gyatso, o décimo quarto Dalai Lama, é o atual líder espiritual do budismo tibetano. Nas últimas décadas, tem desenvolvido uma intensa atividade política e social dirigida à proteção da vida e da cultura do povo tibetano e à promoção universal dos direitos humanos. Gyatso também tem escrito sobre temas filosóficos e éticos e, de forma especial, sobre o diálogo entre ciência e espiritualidade. ${ }^{23-26}$ Laumakis $^{27}$ aponta que uma síntese do pensamento filosófico do Dalai Lama pode ser expressa no estabelecimento de pontes entre uma compreensão acurada da realidade e uma ação moralmente engajada no cotidiano.

Na obra selecionada para análise no presente artigo, Beyond religion: ethics for a whole world, ${ }^{28}$ Gyatso procura sintetizar numa perspectiva secular, isto é, independente de aspectos religiosos do budismo, suas contribuições para as principais questões éticas que observa na atualidade. Para isso, são salientados também aspectos considerados fundamentais para uma compreensão adequada da realidade.

\section{Objetivo}

Apresentado este cenário, o presente estudo propõe-se a explorar possíveis contribuições de uma escola de pensamento oriental, o budismo tibetano, para o MCCP. Este processo teórico exploratório passa por análise da obra de referência do MCCP, Patient-centered Medicine, ${ }^{1}$ de Beyond Religion e de reflexões de lan McWhinney, um dos principais teóricos da MFC contemporânea, que participou ativamente da elaboração e de pesquisas em torno do MCCP. ${ }^{29}$

\section{Método}

Este é um estudo filosófico, no formato de uma revisão narrativa da literatura do tipo opinativa, ${ }^{30} \mathrm{em}$ que é adotada a hermenêutica, na forma apresentada por Gadamer, ${ }^{31}$ como aproximação metodológica. Gadamer propôs a hermenêutica como método adequado à tarefa das ciências humanas: encontrar a 
verdade que está presente nas humanidades - nas artes, na literatura, na filosofia. Esta verdade, todavia, se difere da certeza procurada nas ciências exatas e da natureza. Trata-se de um modo de verdade que reside em elementos da experiência humana, para os quais não se conhecem parâmetros definitivos ou absolutos, e que está associado ao modo como vivemos, fazemos nossas escolhas e elaboramos sentido para elas. Neste caso, a compreensão, tomada como sinônimo de hermenêutica, não segue etapas rígidas, mas implica um movimento de diálogo com o objeto ou texto em investigação, buscando elucidar e respeitar sua intencionalidade original.

Aseguir, serão apresentadas, em seções independentes, uma síntese de elementos de Patient-Centered Medicine e Beyond Religion identificados como potencialmente úteis para tecer conexões teóricas entre o MCCP e a ética secular proposta por Gyatso. Posteriormente, recorremos a textos de McWhinney, em que a compaixão, principal qualidade moral que emerge nestas conexões, é abordada como fundamento para uma prática médica centrada nos relacionamentos humanos.

\section{Uma ética secular como horizonte para as relações humanas}

O Dalai Lama inicia sua proposta de uma ética secular descrevendo a importância e os benefícios sociais trazidos pelos avanços tecnológicos do século XX. Ao mesmo tempo, aponta graves desafios globais contemporâneos da humanidade: a persistência de profundas desigualdades sociais entre e dentro das nações, as guerras e conflitos armados civis e militares, o impacto dos transtornos mentais na vida moderna, entre outros.

Na raiz desta situação, Gyatso identifica uma excessiva valorização de aspectos externos e materiais da vida e a negligência de aspectos que serão denominados valores internos. Por valores internos, entendem-se qualidades que Gyatso considera de apreciação comum pela humanidade como cuidado, zelo, afeição e cordialidade. Tais qualidades podem ser resumidas num princípio fundamental: a compaixão.

"A essência da compaixão é um desejo de aliviar o sofrimento dos outros e promover seu bem-estar. Este é o princípio espiritual do qual todos os outros valores positivos emergem"28 (tradução nossa, p. 9).

Traçada esta análise e apresentada a relevância da compaixão, Beyond Religion passa a ser dedicado a fundamentar e indicar técnicas comportamentais que possam apoiar no desenvolvimento desta qualidade interior. Para compreender como funcionam estas técnicas, é útil notar que, na psicologia budista, cognição e emoção não são vistos separadamente. Emoções são compreendidas como parte de estados mentais; a mente, por sua vez, não é sinônima de capacidade racional. Toda emoção, prazerosa ou incômoda, benéfica ou prejudicial, integra um estado mental, que também inclui também outros elementos psicológicos, como discriminação, volição, atenção, contato e sentimento.

Os estados mentais são ditos prejudiciais quando comprometem o nosso bem-estar a longo prazo, independente de serem, de imediato, prazerosos ou dolorosos. Os estados mentais prejudiciais têm como característica comum uma percepção distorcida da realidade e um obscurecimento de nossa capacidade de discernimento. Por isso, a principal estratégia apontada pelo Dalai Lama para promover estados mentais benéficos, como a compaixão, e evitar estados prejudiciais, como a raiva, o orgulho e o egoísmo, é o cultivo da mente. 
O cultivo da mente ou treinamento mental, apresentado por Gyatso, é um conjunto de práticas contemplativas autoinduzidas para promoção de estados mentais benéficos ou positivos. Como destacado por Wallace e Shapiro, ${ }^{32}$ a psicologia clínica ocidental tem tido como foco principal o diagnóstico e tratamento dos transtornos mentais, enquanto o budismo, desde sua origem, tem utilizado um foco ampliado que destaca o desenvolvimento de técnicas que favorecem estados mentais de profundo bem-estar.

Gyatso explica que este treinamento pode ser traduzido como uma aplicação disciplinada da mente a objetos ou temas benéficos. No budismo, essa aplicação se apresenta em três níveis: escuta ou aprendizado; reflexão; e experiência contemplativa. A escuta e o aprendizado envolvem o contato com estudos prévios dos temas estabelecidos como foco do treinamento. A reflexão envolve uma análise crítica da validade e utilidade destes estudos prévios, esclarecimento de dúvidas e aprendizados complementares. Na experiência contemplativa, são propostas visualizações, usando recursos imaginativos e de percepção sensorial, que conduzem a mente a se estabilizar naquele estado específico que for alvo do treinamento.

Esclarecida esta base metodológica do cultivo mental, Gyatso propõe como aplicá-la ao treinamento da compaixão. São sugeridos dois temas para análise e contemplação. O primeiro é o reconhecimento de nossa humanidade compartilhada, isto é, a observação reflexiva de fatos e experiências do cotidiano que apontam para o fato de que há uma aspiração humana comum à felicidade e a evitar o sofrimento. O segundo tema toca a interdependência como outra qualidade chave de nossa condição humana. As características sociais e culturais do modo de vida humano indicam que um profundo bem-estar físico e mental não pode ser alcançado isoladamente, mas está diretamente vinculado a que outras pessoas também possam experimentar paz e condições protegidas para o seu desenvolvimento.

\section{Medicina centrada na pessoa e cuidado compassivo}

Dos quatro componentes do MCCP, dois, em especial, tocam temas comuns às questões levantadas em Beyond Religion: o primeiro e o quarto. Assim como na ética secular desenvolvida por Gyatso, o ponto de partida do MCCP é o reconhecimento de que uma compreensão integral das questões humanas, entre as quais se encontra a saúde, o adoecimento e a cura, deve incluir uma abordagem não apenas do mundo físico ou externo, mas também de nossos pensamentos, sentimentos e emoções.

O primeiro componente do MCCP - explorar a saúde, a doença e a experiência da doença - sinaliza esta direção. Segundo Stewart, a experiência da doença é a "experiência pessoal e subjetiva de quem está doente: os pensamentos, sentimentos e comportamentos alterados de alguém que se sente doente"1 (p. 36). O profissional médico deve, por meio de técnicas de comunicação verbal e não verbal, explorar estes aspectos do processo de adoecimento de seus pacientes. Ao mesmo tempo, deve manejar recursos para lidar com o sofrimento psíquico e físico a eles associado. "Ser centrado na pessoa requer o equilíbrio entre o subjetivo e o objetivo, um encontro entre mente e corpo"1(p. 4).

Entre os recursos para lidar com a experiência da doença, são destacadas algumas ferramentas como a entrevista motivacional e a elaboração de narrativas pelos pacientes. No entanto, para além destas possibilidades, é apontado como principal recurso para manejo da experiência da doença o quarto componente, a intensificação da relação médico paciente. 
Oferecer reconhecimento, atenção, respeito e parceria emancipatória - traduzidos em afirmações, perguntas, gestos e posturas diante do universo de sentimentos e ideias dos pacientes - é o principal caminho apontado por Stewart para construir uma relação médico-paciente que, em si mesma, seja terapêutica. Uma atitude de cuidado compassivo, cujos núcleos são o reconhecimento do sofrimento do outro e o permanecer integralmente junto a ele nesta experiência, constituem o alicerce da construção do vínculo terapêutico.

Por outro lado, para manifestar esta atitude empática de compreensão e implicação positiva com o sofrimento do outro, o médico deve também manter contato com seu universo subjetivo, com suas próprias emoções, sentimentos e ideias, utilizando-os como suas ferramentas de trabalho, tanto quanto utiliza medicamentos, exames ou procedimentos cirúrgicos.

Para subsidiar este trabalho de autoconsciência - self-awareness - e aprimoramento pessoal, Stewart apresenta algumas possibilidades: os grupos Balint, os grupos de família de origem, os grupos de consciência pessoal propostos por Carl Rogers, a leitura de clássicos da literatura sobre trajetórias de adoecimento e a reflexão sobre a experiência pessoal despertada pelo contato com os pacientes. ${ }^{1}$

Resumindo, o MCCP enfatiza como constituinte da prática médica uma abordagem terapêutica da experiência de doença dos pacientes. Para isso, estabelece, entre diversas possibilidades afins, a intensificação da relação médico-paciente como recurso fundamental. Essa intensificação se dá pelo fortalecimento de uma atitude de cuidado compassivo dos médicos, cuja base é o desenvolvimento de princípios morais e de sentimentos de reconhecimento e engajamento com o sofrimento pessoal dos pacientes. Para a promoção desta atitude, são apontadas algumas estratégias de autoconhecimento individual, em grupo e exploração da própria experiência cotidiana e de exemplos vindos das artes e humanidades.

O MCCP e a abordagem de Gyatso compartilham um objetivo comum: uma compreensão ampliada do fenômeno do sofrimento físico e psíquico humano. O MCCP tem como foco os processos individuais de adoecimento, enquanto, na ética secular, trata-se de problemas coletivos derivados de questões sociais contemporâneas. Em ambos os casos, identifica-se, como diagnóstico inicial, a necessidade de reconhecer e valorizar aspectos internos e subjetivos da experiência humana. Num segundo momento, também em ambos os trabalhos, encontra-se o desenvolvimento da compaixão como uma possibilidade para lidar com esta dimensão menos física e mais subjetiva do nosso sofrimento comum.

\section{Compaixão e medicina de família}

Um aprofundamento dessas conexões pode ser alcançado por meio das reflexões de lan McWhinney sobre o método clínico centrado na pessoa e sobre a medicina de família. Uma significativa contribuição para a compreensão do papel da compaixão no método clínico centrado na pessoa é desenvolvida por este autor. Para ele, a transformação do método clínico está vinculada a um amplo processo de mudança na Medicina e na atitude de seus praticantes, que exige "uma transformação no que significa ser médico, uma forma diferente de pensar sobre saúde e doença, e uma redefinição do conhecimento médico"33 (p. 47).

Ao definir a medicina de família (MFC) como disciplina singular, McWhinney aponta, como seu campo, o compromisso irrestrito de cuidado de um médico com um grupo de pessoas. ${ }^{34}$ Assim, a MFC se define por meio de relações e não por conhecimentos ou técnicas (doenças ou tecnologias de intervenção) como 
outras especialidades médicas. Ao se definir por meio de relações, descobre-se a necessidade de novas categorias de valoração do conhecimento e novas formas de pensar.

Para compreender e manejar relacionamentos entre pessoas, não são suficientes as abstrações e generalizações de modelos teóricos explicativos, uma razão instrumental e desengajada, comum no modelo científico moderno. Esta tarefa demanda um saber que surja da experiência concreta e única de cada relação e que, inevitavelmente, engaja intelecto e emoções. Portanto, o reconhecimento e manejo de emoções e sentimentos, de médicos e pacientes, ocupam lugar central na medicina de família. "Quando falamos de relacionamentos, temos de falar das emoções, são as emoções que constroem ou destroem os relacionamentos"35 (tradução nossa, p. 193).

Também fica claro que, para abordar relações, o modelo do corpo humano como máquina desconectada de aspectos da subjetividade (o dualismo mente-corpo) e a separação entre razão e emoção são inadequados. Não estamos lidando com máquinas passivas diante de eventuais necessidades de reparo, mas com organismos que possuem processos de autoorganização e autorregeneração diante de traumas e desequilíbrios. ${ }^{36}$

Nesta perspectiva, designada por McWhinney como organicista, pode ser aplicada à categoria de cura como o reestabelecimento de um novo estado de equilíbrio pelos organismos. Neste processo, os médicos assumem a tarefa de curadores. "Um curador é alguém que caminha conosco, não nos julgando, mas revelando o que é mais valioso em nós e apontando na direção de um significado para nossa dor interior"37 (tradução nossa, p. 314)

Tanto para lidar com as emoções, sentimentos e humores quanto para exercer o papel de curadores, McWhinney destaca uma habilidade chave: a escuta ativa e atenta dos pacientes. "É escutar não apenas com nossos ouvidos, mas todas as nossas faculdades, especialmente com um coração aberto" 38 (tradução nossa, p. 435). Esta habilidade é fundamentada numa qualidade ou estado de atenção, denominado ora como compaixão ${ }^{37}$ ora como agape, ${ }^{38}$ ou amor impessoal não condicional. "Se pudermos atingir este estado de abertura, descobrimos que nossas respostas aos pacientes fluem naturalmente dessa fonte interior" 38 (tradução nossa, p. 435).

Contudo, o desenvolvimento desse estado interno compassivo tem um obstáculo principal: nossas emoções negativas. ${ }^{33,38}$ Emoções negativas são estados emocionais egoístas não reconhecidos e não examinados, como medo, desamparo, raiva, culpa, autocentramento, que fazem com que nos afastemos do sofrimento dos outros e vejamos apenas nossas necessidades emocionais. O principal caminho para lidar com as emoções negativas, segundo McWhinney, é o autoconhecimento. "Nosso envolvimento deve estar livre de apegos autocentrados. Para alcançar este envolvimento desapegado, necessitamos de autoconhecimento: inteligência emocional, paz mental"37 (tradução nossa, p. 315).

Conexões mais evidentes entre as considerações de Gyatso e de McWhinney podem, assim, ser estabelecidas. O desenvolvimento da compaixão tem papel central, e não apenas adjuvante, para as mudanças pretendidas por ambos em seus campos de análise. Também uma aproximação da compaixão e das emoções com tratamentos epistemológicos não dualistas, que partem de categorias mais holistas como "estado mental", "experiência" e "relações", que integram corpo, intelecto e emoções, é sugerida nos dois casos. Os dois autores coincidem em apontar emoções negativas, autocentradas, como principal obstáculo para o desenvolvimento da compaixão. 
A principal diferença se encontra nas propostas de como lidar com essas emoções. McWhinney apresenta a direção do autoconhecimento, do reconhecimento pessoal dessas emoções negativas, e deixa algumas sinalizações, sem muitos detalhamentos, como "paz mental", "inteligência emocional", orientações da obra de Balint e a observação individual da prática clínica por um mentor de confiança, como caminhos para este autoconhecimento. Sua análise se aproxima mais da ênfase da psicologia ocidental moderna, que procura estratégias para identificar e retirar de cena as emoções negativas.

Propomos que os apontamentos de Gyatso para desenvolvimento da compaixão sejam vistos como complementares. A ênfase do Dalai Lama é de que esses estados mentais podem ser promovidos também por técnicas comportamentais autoinduzidas que cultivem qualidades positivas, especificamente, a compaixão. Estas técnicas são mais dirigidas a colocar em cena a compaixão do que retirar as emoções autocentradas, ainda que este seja um efeito indireto também alcançado.

\section{Considerações finais}

O desenvolvimento de estratégias de manejo dos aspectos psicossociais do profissional de saúde envolvido na relação com o paciente interessa à Medicina contemporânea, sobremaneira como alicerce para um método clínico centrado na pessoa. O presente trabalho buscou ampliar o leque de possibilidades para conduzir este manejo. Apresentamos a fundamentação epistemológica, ética e psicológica de métodos originados no budismo, na forma apresentados pelo Dalai Lama, que podem ser usados com esta finalidade.

Optou-se, em coerência com a abordagem hermenêutica, pela ênfase nas reflexões sobre valores e discursos que subsidiam os métodos, mais do que em descrições detalhadas das técnicas. A convergência entre a abordagem do universo emocional proposta por McWhinney e aquela proposta por Gyatso é um dos resultados particularmente significativos deste estudo. Demonstra a possibilidade de encontro entre tradições culturais distintas na busca por respostas às inquietações humanas diante do sofrimento. Aponta também para um horizonte de inclusão, respeito e pluralidade cultural para tratar de aspectos humanos, e por isso, não normatizáveis, da Medicina: como lidar com as emoções dos pacientes? Dos colegas de trabalho? Como lidar com minhas emoções?

A dificuldade de encontrar respostas únicas para estas questões, muitas vezes, nos afasta do seu debate, seja na formação, seja na pesquisa médica. Voltando-se para essa dificuldade, o presente trabalho procura se situar na agenda proposta por McWhinney, ${ }^{39}$ em que o desenvolvimento de insight e consciência é um dos três tipos de conhecimento médico, tão importante quanto as informações e as habilidades clínicas. Da mesma forma que Russell aponta acom relação à filosofia em geral, entendemos que, também para o campo particular do insight e da consciência, o mais importante não são as repostas às questões que mobilizam nossa investigação, mas as próprias questões em si, que "alargam nossa concepção do que é possível, enriquecem nossa imaginação intelectual e diminuem a segurança dogmática que fecha a mente à especulação"40 (tradução nossa, p. 442).

Se a centralidade da pessoa é uma das características desejada para a Medicina contemporânea, o desenvolvimento da compaixão como uma atitude fundamental deve ocupar lugar importante na formação e na prática médica. Tal desenvolvimento, segundo as teorias que aqui visitamos, está vinculado ao 
treinamento de habilidades reflexivas e psicológicas que promovam, especificamente, a atitude compassiva. Adotar esse caminho e investigar seus determinantes, seus pormenores e suas consequências são as próximas tarefas de uma agenda de pesquisa dele derivada.

\section{Referências}

1. Stewart M, Brown JB, Weston WW, McWhinney IR, McWilliam CL, Freeman TR. Patient-centered medicine. Transforming the clinical method. 3rd ed. New York: Radcliffe Publishing; 2014.

2. McWhinney IR. A evolução do método clínico. In: Stewart M, Brown JB, Weston WW, Mc Whinney IA, McWilliam CL, Freeman TR. Medicina centrada na pessoa: transformando o método clínico. 2a ed. Porto Alegre: Artmed; 2010. p.35-48.

3. Wilson H. Becoming 'patient-centred': a review. N Z Fam Pract. 2008;35(3):164-70.

4. Lopes JMC. Consulta e abordagem centrada na pessoa. In: Gusso G, Lopes JMC, orgs. Tratado de medicina de família e comunidade: princípios, formação e prática. Porto Alegre: Artmed; 2012. p.113-23.

5. Castro RCL. Evidências dos benefícios da medicina centrada na pessoa. In: SBMFC, Augusto DK, Umpierre RN, orgs. PROMEF: Programa de atualização em medicina de família e comunidade. Porto Alegre: Artmed/Panamericana; 2013. p.9-50.

6. Borrell-Carrió F, Suchman AL, Epstein RM. The biopsychosocial model 25 years later: principles, practice, and scientific inquiry. Ann Fam Med. 2004;2(6):576-82. DOI: http://dx.doi.org/10.1370/afm.245

7. Camargo Júnior KR. O paradigma clínico-epidemiológico ou biomédico. Rev Bras Hist Ciên. 2013;6(2):183-95.

8. Guedes CR, Nogueira MI, Camargo Júnior KR. A subjetividade como anomalia: contribuições epistemológicas para a crítica do modelo biomédico. Ciênc Saúde Coletiva. 2006;11(4):1093-103. DOI: http://dx.doi.org/10.1590/S141381232006000400030

9. Little P, Everitt H, Williamson I, Warner G, Moore M, Gould C, et al. Preferences of patients for patient centred approach to consultation in primary care: observational study. BMJ. 2001;322(7284):468-72. PMID: 11222423 DOI: http://dx.doi. org/10.1136/bmj.322.7284.468

10. Stewart M. Reflections on the doctor-patient relationship: from evidence and experience. Br J Gen Pract. 2005;55(519):793801.

11. Novack DH, Suchman AL, Clark W, Epstein RM, Najberg E, Kaplan C. Calibrating the physician. Personal awareness and effective patient care. Working Group on Promoting Physician Personal Awareness, American Academy on Physician and Patient. JAMA. 1997;278(6):502-9. PMID: 9256226 DOI: http://dx.doi.org/10.1001/jama.1997.03550060078040

12. Churchill LR, Schenck D. Healing skills for medical practice. Ann Intern Med. 2008;149(10):720-4. PMID: 19017590 DOI: http://dx.doi.org/10.7326/0003-4819-149-10-200811180-00006

13. Judd F, Weissman M, Davis J, Hodgins G, Piterman L. Interpersonal counselling in general practice. Aust Fam Physician. 2004;33(5):332-7. PMID: 15227863

14. Aung SK. Loving kindness: the essential Buddhist contribution to primary care. Hum Health Care Int. 1996;12(2):E12.

15. Neher JO, Brown N. Teaching tips for clinical faculty from the Zen tradition. Fam Med. 1997;29(7):513-6. PMID: 9232415

16. Garrett RE, Klinkman M, Post L. If you meet Buddha on the road, take a genogram: Zen and the art of family medicine. Fam Med. 1987;19(3):225-6.

17. Neighbour R. The inner consultation: how to develop an effective and intuitive consulting style. 2nd ed. Oxford: Radcliffe Medical Press; 2004.

18. Goyal M, Singh S, Sibinga EM, Gould NF, Rowland-Seymour A, Sharma R, et al. Meditation programs for psychological stress and well-being: a systematic review and meta-analysis. JAMA Intern Med. 2014;174(3):357-68. DOI: http://dx.doi. org/10.1001/jamainternmed.2013.13018

19. Ost LG. The efficacy of Acceptance and Commitment Therapy: an updated systematic review and meta-analysis. Behav Res Ther. 2014;61:105-21. PMID: 25193001 DOI: http://dx.doi.org/10.1016/j.brat.2014.07.018 
20. Hayes SC. Buddhism and acceptance and commitment therapy. Cogn Behav Pract. 2002;9(1):58-66. DOI: http://dx.doi. org/10.1016/S1077-7229(02)80041-4

21. Fung K. Acceptance and Commitment Therapy:Western adoption of Buddhist tenets? Transcult Psychiatry. 2015;52(4):561 76.

22. Samuel G. The contemporary mindfulness movement and the question of nonself. Transcult Psychiatry. 2015;52(4):485500. DOI: http://dx.doi.org/10.1177/1363461514562061

23. Bhattacharjee Y. Neuroscience. Neuroscientists welcome Dalai Lama with mostly open arms. Science. 2005;310(5751):1104. PMID: 16293731 DOI: http://dx.doi.org/10.1126/science.310.5751.1104

24. Gyatso T. The contribution of the Tibetan Medical System to global health. Forsch Komplementmed. 2006;13 Suppl 1:VI. DOI: http://dx.doi.org/10.1159/000090995

25. Knight J. Religion and science: Buddhism on the brain. Nature. 2004;432(7018):670. PMID: 15592378 DOI: http://dx.doi. org/10.1038/432670a

26. Dalai Lama. The universe in a single atom: The convergence of science and spirituality. New York: Harmony; 2006.

27. Laumakis SJ. An introduction to Buddhist philosophy. Cambridge University Press: New York; 2008.

28. Dalai Lama. Beyond Religion: ethics for a whole world. London: Rider; 2011.

29. Scherger JE. Remembering lan McWhinney. Fam Med. 2013;45(8):539-40.

30. Green BN, Johnson CD, Adams A. Writing narrative literature reviews for peer-reviewed journals: secrets of the trade. J Chiropr Med. 2006;5(3):101-17. DOI: http://dx.doi.org/10.1016/S0899-3467(07)60142-6

31. Gadamer HG. Verdade e método I: traços fundamentais de uma hermenêutica filosófica. 9a ed. Petrópolis: Vozes; 2008.

32. Wallace BA, Shapiro SL. Mental balance and well-being: building bridges between Buddhism and Western psychology. Am Psychol. 2006;61(7):690-701. PMID:17032069 DOI: http://dx.doi.org/10.1037/0003-066X.61.7.690

33. McWhinney IR. A evolução do método clínico. In: Stewart M, Brown JB, Weston WW, McWhinney IA, McWilliam CL, Freeman TR. Medicina centrada na pessoa: transformando o método clínico. 2a ed. Porto Alegre: Artmed; 2010. p.35-48.

34. McWhinney IR, Freeman T. A textbook of family medicine. 3rd ed. New York: Oxford University Press; 2009.

35. McWhinney IR. The importance of being different. Part 1: The marginal status of family medicine. Can Fam Physician. 1997;43:193-5.

36. McWhinney IR. The importance of being different. Part 2: Transcending the mind-body fault line. Can Fam Physician. 1997;43:404-6.

37. McWhinney IR. Being a general practitioner: what it means. Prim Care. 2001;1:309-16.

38. McWhinney IR. William Pickles Lecture 1996. The importance of being different. Br J Gen Pract. 1996;46(408):433-6.

39. McWhinney IR. Family medicine in perspective. N Engl J Med. 1975;293(4):176-81. DOI: http://dx.doi.org/10.1056/ NEJM197507242930405

40. Berkeley JS. Towards a philosophy of family medicine. J R Coll Gen Pract. 1983;33(252):442-4. PMID: 6887115 